\title{
Multivariate Spatial IV Regression
}

\author{
Marcus G. Lavagnole Nascimento* \\ Carlos A. Abanto-Valle* \\ Mario Jorge MEndonçA**
}

\begin{abstract}
In this paper, a Multivariate Spatial Regression model with Endogenous Variables is proposed. In order to deal with endogeneity and spatial dependence, the instrumental variables (IV) methodology and an autoregressive spatial structure, frequently used in econometric applications, are implemented. A Bayesian inference procedure based on simulation schemes designed to obtain samples from the posterior distribution of model parameters is developed. Finally, the methodology is illustrated through an application to the impact of broadband access on the economic sectors.
\end{abstract}

Keywords: Bayesian inference, endogenous variables, instrumental variables, broadband access

JEL Codes: C01, C11, C26

\section{Introduction}

Simultaneous equation models (SEMs) and Instrumental Variables (IV) constitute powerful tools in statistics and econometrics. The IV regression model, in special, is a common method for calculating treatment effects for endogenous regressors and has been the focus of a variety of studies which explored a myriad of methods, listed in Lopes and Polson (2014), including: Bayesian approach (Zellner, 1971), Bayes-Stein shrinkage (Zellner \& Vandaele, 1975), decision-theoretic methods (Chamberlain, 2007), methods of the moments (Zellner, Tobias, \& Ryu, 1997), semiparametric Dirichlet mixtures (Conley, Hansen, McCulloch, \& Rossi, 2008) and Monte Carlo simulation (Zellner, Bauwens, \& van Dijk, 1988), just to

Submitted on 19 March 2018; Reviewed on 03 September 2018

* Departamento de Métodos Estatísticos, Universidade Federal do Rio de Janeiro (DME/UFRJ), Rio de Janeiro, RJ, CEP 21945-970, Caixa Postal 68530, Brazil.

** Instituto de Pesquisa Econômica Aplicada (Ipea), Brazil.

-marcus@dme.ufrj.br 
mention a few. By the other hand, Lindley and El-Sayyad (1968) and Kleibergen and Zivot (2003) turned its attention to comparisons between Bayesian and classical approaches.

However, most of the developments in the area deal with the case in what the response variable and the instrumental variable are univariate. In this paper, a particular case of the multivariate spatial regression models suggested by Gamerman and Moreira (2004) is extended to the context of endogenous variables. To that end, based on extensions of the ideas applied by Rossi, Allenby, and McCulloch (2005), Bayesian inference is performed in a multivariate setup for the response variable and the instrumental variable.

The remainder of the paper is organized as follows. Section 2 outlines the basic IV regression setup and introduces the multivariate spatial regression model (Gamerman \& Moreira, 2004). In section 3, the multivariate spatial IV regression is developed and the Bayesian inference procedure is described. In section 4, a study with synthetic data is realized and an application focused on measuring the impact of broadband expansion on Brazilian counties is executed. Finally, in section 5, some concluding remarks are made.

\section{Preliminaries}

\subsection{Regression Model}

The follow basic considerations that will be introduced in this section are based on Lancaster (2004). Let $y_{i}$ be a response variable and $x_{i}$ an endogenous covariate, $i=1, \ldots, n$, as stated in the system of equations

$$
\begin{aligned}
& x_{i}=\gamma+\delta z_{i}+\varepsilon_{1 i} \\
& y_{i}=\alpha+\beta x_{i}+\varepsilon_{2 i},
\end{aligned}
$$

where $z_{i}$ is an instrument related to $x_{i}$ but independent on $\varepsilon_{1 i}$. It is assumed that $\boldsymbol{\varepsilon}_{i}=\left(\varepsilon_{1 i}, \varepsilon_{2 i}\right)^{\prime}$ are independent and identically distributed with $\varepsilon_{i} \sim \mathcal{N}_{2}(0, \boldsymbol{\Sigma})$, where $\mathcal{N}_{m}$ denotes a $m$-dimensional multivariate normal distribution. In this case, the covariance matrix has the main diagonal equals to $\sigma_{11}$ and $\sigma_{22}$, the off-diagonal equals to $\sigma_{12}=\rho\left(\sigma_{11} \sigma_{22}\right)^{1 / 2}$ in which $\rho$ is the correlation between $x_{i}$ and $y_{i}$.

The fundamental difference between the above system of equations to a standard bivariate regression is the possible correlation between the error terms 
$\varepsilon_{1 i}$ and $\varepsilon_{2 i}$, therefore, between $x_{i}$ and $\varepsilon_{2 i}$. It induces a well-known "endogeneity" bias when estimating $\beta$ from equation (2), it means, the information of $x_{i}$ that is correlated with $\varepsilon_{2 i}$ should not be used when estimating about the regression parameter $\beta$.

The system of equations described by (1) and (2) is denominated the structural form because it defines how each variable is connected to the others according to the theory. However, usually, the inference process is realized through the reduced form written as follows:

$$
\begin{aligned}
& x_{i}=\gamma+\delta z_{i}+v_{1 i} \\
& y_{i}=\alpha^{*}+\beta \delta z_{i}+v_{2 i},
\end{aligned}
$$

in which $\alpha^{*}=\alpha+\beta \gamma, v_{1 i}=\varepsilon_{1 i}$, and $v_{2 i}=\varepsilon_{2 i}+\beta \varepsilon_{1 i}$. Differently from the structural form, the equations (3) and (4) are linear regressions due to the independence between $z_{i}$ and the errors. In this case, parameter estimation is done through the conventional procedures applied to regression models and $\beta$ is recovered as the ratio of $\beta \delta$ and $\delta$.

\subsection{Multivariate Spatial Regression}

Gamerman and Moreira (2004) developed a Bayesian methodology to multivariate spatial regression models applied to areal data sets. Among these models, it will be underlined a class that imposes the spatial restriction directly through the observation equation, more specifically, those ones that include in the mean an autoregressive spatial component which considers a spatial localization. This autoregressive spatial component is frequently used in econometrics applications and can be implemented on the fixed part of the model (Anselin, 1988), on the random part (Pace, Barry, Gilley, \& Sirmans, 2000), and on both ones (Anselin, 1988).

Let $\mathbf{y}_{i}=\left(y_{i 1}, \ldots, y_{i q}\right)^{\prime}$ be a $q$-dimensional response variable vector and $\mathbf{x}_{i}^{*}=\left(x_{i 1}^{*}, \ldots, x_{i r}^{*}\right)^{\prime}$, an $r$-dimensional exogenous variable vector. The multivariate regression can be generalized incorporating a spatial dependence via

$$
\begin{aligned}
\mathbf{y A} & =\mathbf{W}_{1} \mathbf{y} \boldsymbol{\phi}+\mathbf{x}^{*} \boldsymbol{\beta}+\mathbf{u} \\
\mathbf{u} & =\mathbf{W}_{2} \mathbf{u} \psi+\boldsymbol{\epsilon}, \quad \boldsymbol{\epsilon} \sim \mathcal{N}_{n \times q}\left(0, \mathbf{I}_{n}, \boldsymbol{\Lambda}\right),
\end{aligned}
$$

in which $\mathbf{y}=\left(\mathbf{y}_{1}, \ldots, \mathbf{y}_{n}\right)^{\prime}$ and $\mathbf{x}^{*}=\left(\mathbf{x}_{1}^{*}, \ldots, \mathbf{x}_{n}^{*}\right)^{\prime}$. Additionally, $\mathbf{A}, \boldsymbol{\Lambda}, \boldsymbol{\phi}$, 
and $\boldsymbol{\psi}$ are $q \times q$ matrices but $\boldsymbol{\Lambda}$ is diagonal. $\mathcal{N}_{a \times b}(\cdot, \cdot, \cdot)$ denote the matrix normal distribution. In particular, the matrices $\mathbf{W}_{1}$ and $\mathbf{W}_{2}$ are neighborhood matrices representing a neighborhood structure and may be equal to $\mathbf{W}$. Hence, $\mathbf{W}=\left(w_{i j}\right), w_{i j}$ representing the neighborhood between sites $i$ and $j$, is a matrix in which $w_{i j} \neq 0$ for neighbors sites and $w_{i j}=0$ otherwise. The standard value for $w_{i j}$ is $1 / m_{i}, m_{i}$ is the number of neighbors of site $i$.

Gamerman and Moreira (2004) describe a special case of the model expressed by equations (5) and (6) when $\phi$ and $\boldsymbol{\psi}$ is given by diagonal forms with respective entries $\phi_{k k}$ and $\psi_{k k}, k=1, \ldots, q$. The $\phi_{k k}$ 's and $\psi_{k k}$ 's consist on the autoregressive coefficients of the observations and of the errors. Assuming $\mathbf{A}=\mathbf{I}_{q}$ and diagonal forms for $\boldsymbol{\phi}$ and $\boldsymbol{\psi}$, the equations (5) and (6) can written as follows

$$
\begin{aligned}
& \mathbf{y}^{k}=\phi_{k k} \mathbf{W}_{1} \mathbf{y}^{k}+\mathbf{x}^{*} \boldsymbol{\beta}_{k}+\mathbf{u}^{k} \\
& \mathbf{u}^{k}=\psi_{k k} \mathbf{W}_{2} \mathbf{u}^{k}+\boldsymbol{\epsilon}^{k}, \quad \boldsymbol{\epsilon}^{k} \sim \mathcal{N}_{n}\left(0, \mathbf{I}_{n} \sigma_{k k}^{2}\right),
\end{aligned}
$$

where $\mathbf{y}^{k}=\left(y_{11}, \ldots, y_{n 1}\right)^{\prime}$ and the same structure is applied for the other vectors. The above equations seem to indicate independence between the components of the response. Nevertheless, the response is correlated because the errors $\boldsymbol{\epsilon}^{k}$ 's are not independent.

\section{Multivariate IV Spatial Regression Model}

\subsection{The Model}

Supported by the methodology proposed by Gamerman and Moreira (2004) and by the ideas introduced by (Rossi et al., 2005), in this section, a Bayesian framework for a multivariate spatial regression model with endogenous variables is developed. The structural form of the linear IV regression described by equations (1) and (2) is assumed and generalized, the assumptions made on equations (7) and (8) are considered with the restriction that the autoregressive spatial component is applied in the fixed part of the model. Thence,

$$
\begin{aligned}
& \mathbf{x}_{i}=\boldsymbol{\gamma}+\boldsymbol{\delta} \mathbf{z}_{i}+\varepsilon_{1 i} \\
& \mathbf{y}_{i}=\boldsymbol{\phi}(\mathbf{W} \mathbf{y})_{i}^{\prime}+\boldsymbol{\beta}_{1} \mathbf{x}_{i}^{*}+\boldsymbol{\beta}_{2} \mathbf{x}_{i}+\varepsilon_{2 i}
\end{aligned}
$$

in which $\mathbf{x}_{i}=\left(x_{i 1}, \ldots, x_{i p}\right)^{\prime}$ is a $p$-dimensional endogenous variable vector, $\mathbf{z}_{i}=\left(z_{i 1}, \ldots, z_{i l}\right)^{\prime}$ is a $l$-dimensional instrumental variable vector, and $(\mathbf{W y})_{i}$ 
is the $i$-th line of the $\mathbf{W y}$ matrix product. In this case, it is assumed that $\boldsymbol{\varepsilon}_{i}=\left(\boldsymbol{\varepsilon}_{1 i}, \boldsymbol{\varepsilon}_{2 i}\right)$ are independent and identically distributed with $\boldsymbol{\varepsilon}_{i} \sim \mathcal{N}_{(p+q)}(0, \boldsymbol{\Sigma})$ and the covariance matrix has the main diagonal equals to the block diagonal $\boldsymbol{\Sigma}_{11}$ and $\boldsymbol{\Sigma}_{22}$ and the off-diagonal, by the block diagonal $\boldsymbol{\Sigma}_{12}=\boldsymbol{\Sigma}_{21}^{\prime}$. Thus, the joint density of $\mathbf{y}$ and the instrumental variable $\mathbf{x}$ is

$$
f\left(\mathbf{y}, \mathbf{x} \mid \phi, \mathbf{x}^{*}, \boldsymbol{\beta}_{1}, \boldsymbol{\beta}_{2}, \boldsymbol{\gamma}, \mathbf{z}, \boldsymbol{\delta}, \Sigma\right)=\prod_{i=1}^{n} f\left(\mathbf{y}_{i}, \mathbf{x}_{i} \mid \boldsymbol{\phi}, \mathbf{x}_{i}^{*}, \boldsymbol{\beta}_{1}, \boldsymbol{\beta}_{2}, \boldsymbol{\gamma}, \mathbf{z}_{i}, \boldsymbol{\delta}, \Sigma\right)
$$

where $\mathbf{x}=\left(\mathbf{x}_{1}, \ldots, \mathbf{x}_{n}\right)^{\prime}$ and $\mathbf{z}=\left(\mathbf{z}_{1}, \ldots, \mathbf{z}_{n}\right)^{\prime}$.

\subsection{Bayesian Inference}

Performing a Bayesian analysis, the first step is to select the priors distributions. Excepting the parameter $\phi$, extensions of the conjugated priors specified by Rossi et al. (2005) were chosen, it means, $(\boldsymbol{\gamma}, \boldsymbol{\delta})^{\prime} \mid \boldsymbol{\Sigma}_{1 \mid 2} \sim \mathcal{N}_{(l+1) \times p}\left(\boldsymbol{d}_{0}, \mathbf{D}_{0}, \boldsymbol{\Sigma}_{1 \mid 2}\right)$, $\left(\boldsymbol{\beta}_{1}, \boldsymbol{\beta}_{2}\right)^{\prime} \mid \boldsymbol{\Sigma}_{2 \mid 1} \sim \mathcal{N}_{(r+p) \times q}\left(\mathbf{b}_{0}, \mathbf{B}_{0}, \boldsymbol{\Sigma}_{2 \mid 1}\right)$, and $\boldsymbol{\Sigma} \sim \mathcal{I} \mathcal{W}\left(\nu_{0}, \boldsymbol{\Sigma}_{0}\right)$, in which $\boldsymbol{d}_{0}$, $\mathbf{D}_{0}, \mathbf{b}_{0}, \mathbf{B}_{0}, \nu_{0}, \boldsymbol{\Sigma}_{0}$ are known hyperparameters, $\boldsymbol{\Sigma}_{1 \mid 2}=\boldsymbol{\Sigma}_{11}-\boldsymbol{\Sigma}_{12} \boldsymbol{\Sigma}_{22}^{-1} \boldsymbol{\Sigma}_{21}$ and $\boldsymbol{\Sigma}_{2 \mid 1}=\boldsymbol{\Sigma}_{22}-\boldsymbol{\Sigma}_{21} \boldsymbol{\Sigma}_{11}^{-1} \boldsymbol{\Sigma}_{12}$. IW $(\cdot, \cdot)$ denote the inverse Wishart distribution. Considering $\boldsymbol{\phi}$, as argued by Gamerman and Moreira (2004), there is no obvious option for the parameter prior distribution, however, as it is being adopted $\phi$ diagonal, the authors affirm being reasonable to assume an i.i.d. form, thus

$$
p(\phi)=\prod_{k=1}^{q} p\left(\phi_{k k}\right)
$$

Under the restriction of $\phi_{k k} \in(0,1)$, a natural option was the non-informative prior distribution $\phi_{k k} \sim U(0,1)$, where $U(a, b)$ denotes the uniform distribution on the interval $(a, b)$.

The joint posterior density of the parameters can be written as

$$
p\left(\boldsymbol{\theta} \mid \mathbf{y}, \mathbf{x}^{*}, \mathbf{x}, \mathbf{z}\right) \propto\left[\prod_{i=1}^{n} f\left(\mathbf{y}_{i}, \mathbf{x}_{i} \mid \boldsymbol{\phi}, \mathbf{x}_{i}^{*}, \boldsymbol{\beta}_{1}, \boldsymbol{\beta}_{2}, \boldsymbol{\gamma}, \mathbf{z}_{i}, \boldsymbol{\delta}, \Sigma\right)\right] p(\boldsymbol{\theta}),
$$

where $\boldsymbol{\theta}=\left(\boldsymbol{\gamma}, \boldsymbol{\delta}, \boldsymbol{\beta}_{1}, \boldsymbol{\beta}_{2}, \boldsymbol{\Sigma}, \boldsymbol{\phi}\right)$ and $p(\boldsymbol{\theta})=p\left(\boldsymbol{\gamma}, \boldsymbol{\delta} \mid \boldsymbol{\Sigma}_{1 \mid 2}\right) p\left(\boldsymbol{\beta}_{1}, \boldsymbol{\beta}_{2} \mid \boldsymbol{\Sigma}_{2 \mid 1}\right) p(\boldsymbol{\Sigma}) p(\boldsymbol{\phi})$ as prior independence is assumed for some particular parameters. Thus, the full 
conditionals of the parameters are

$$
\begin{aligned}
p(\boldsymbol{\gamma}, \boldsymbol{\delta} \mid \cdots) \propto\left[\prod_{i=1}^{n} f\left(\mathbf{x}_{i} \mid \mathbf{y}_{i}, \boldsymbol{\phi}, \mathbf{x}_{i}^{*}, \boldsymbol{\beta}_{1}, \boldsymbol{\beta}_{2}, \boldsymbol{\gamma}, \mathbf{z}_{i}, \boldsymbol{\delta}, \boldsymbol{\Sigma}\right)\right] p\left(\boldsymbol{\gamma}, \boldsymbol{\delta} \mid \boldsymbol{\Sigma}_{1 \mid 2}\right), \\
p\left(\boldsymbol{\beta}_{1}, \boldsymbol{\beta}_{2} \mid \cdots\right) \propto\left[\prod_{i=1}^{n} f\left(\mathbf{y}_{i} \mid \boldsymbol{\phi}, \mathbf{x}_{i}^{*}, \boldsymbol{\beta}_{1}, \boldsymbol{\beta}_{2}, \mathbf{x}_{i}, \boldsymbol{\gamma}, \mathbf{z}_{i}, \boldsymbol{\delta}, \boldsymbol{\Sigma}\right)\right] p\left(\boldsymbol{\beta}_{1}, \boldsymbol{\beta}_{2} \mid \boldsymbol{\Sigma}_{2 \mid 1}\right), \\
p(\boldsymbol{\Sigma} \mid \cdots) \propto\left[\prod_{i=1}^{n} f\left(\mathbf{y}_{i}, \mathbf{x}_{i} \mid \boldsymbol{\phi}, \mathbf{x}_{i}^{*}, \boldsymbol{\beta}_{1}, \boldsymbol{\beta}_{2}, \boldsymbol{\gamma}, \mathbf{z}_{i}, \boldsymbol{\delta}, \boldsymbol{\Sigma}\right)\right] p(\boldsymbol{\Sigma}), \\
p(\boldsymbol{\phi} \mid \cdots) \propto\left[\prod_{i=1}^{n} f\left(\mathbf{y}_{i} \mid \boldsymbol{\phi}, \mathbf{x}_{i}^{*}, \boldsymbol{\beta}_{1}, \boldsymbol{\beta}_{2}, \mathbf{x}_{i}, \boldsymbol{\gamma}, \mathbf{z}_{i}, \boldsymbol{\delta}, \boldsymbol{\Sigma}\right)\right] p(\boldsymbol{\phi}) .
\end{aligned}
$$

Additional details about the full conditionals are available in Appendix A. In furtherance of making Bayesian analysis feasible for parameter estimation in the Multivariate Spatial IV Regression model, random samples from the posterior distributions of $\left(\boldsymbol{\gamma}, \boldsymbol{\delta}, \boldsymbol{\beta}_{1}, \boldsymbol{\beta}_{2}, \boldsymbol{\Sigma}, \boldsymbol{\phi}\right)$ given $\mathbf{y}, \mathbf{x}^{*}, \mathbf{x}, \mathbf{z}$ are drawn through Monte Chain Monte Carlo simulation methods. Algorithm 1 describes the sampling scheme from the full conditionals distributions of the parameters.

Algorithm 1: MCMC for Multivariate Spatial IV Regression models.

[1] Set $t=1$ and get starting values for $\boldsymbol{\gamma}^{(0)}, \boldsymbol{\delta}^{(0)}, \boldsymbol{\beta}_{1}^{(0)}, \boldsymbol{\beta}_{2}^{(0)}, \boldsymbol{\Sigma}^{(0)}$, and $\boldsymbol{\phi}^{(0)}$;

[2] Sample $\left(\boldsymbol{\gamma}^{(t)}, \boldsymbol{\delta}^{(t)}\right)$ from $p(\boldsymbol{\gamma}, \boldsymbol{\delta} \mid \cdots)$;

[3] Sample $\left(\boldsymbol{\beta}_{1}^{(t)}, \boldsymbol{\beta}_{2}^{(t)}\right)$ from $p\left(\boldsymbol{\beta}_{1}, \boldsymbol{\beta}_{2} \mid \cdots\right)$;

[4] Sample $\boldsymbol{\Sigma}^{(t)}$ from $p(\boldsymbol{\Sigma} \mid \cdots)$;

[5] Sample $\phi^{(t)}$ from $p(\phi \mid \cdots)$;

[6] Set $t=t+1$ and repeat the steps $2-5$ until convergence is achieved.

\section{Application}

\subsection{Synthetic Data}

The performance of the above algorithm is illustrated with a synthetic data set, samples from the multivariate spatial IV regression, introduced by equations (9) and (10), are artificially generated and it is verified if the proposed methodology is capable to recover the original parameters.

For the estimation process, the hyperparameters set is defined as: $\boldsymbol{d}_{0}=\mathbf{0}_{(3 \times \mathbf{2})}$, $\mathbf{D}_{0}=\mathbf{I}_{3} 10^{3}, \mathbf{b}_{0}=\mathbf{0}_{(5 \times \mathbf{3})}, \mathbf{B}_{0}=\mathbf{I}_{5} 10^{3}, \nu_{0}=6$ e $\boldsymbol{\Sigma}_{0}=\mathbf{I}_{5}$, corresponding to 
non-informative priors specification. 50,000 iterations of the algorithm MCMC were executed, in which the first 20,000 were discarded like the warming period, and then the following 30,000 were saved. In order to reduce the autocorrelation within the successive values of the simulated chain, it was required a thin equals to 30 . Finally, the estimations a posteriori were performed based on a sample size of 1,000 .

Tables 4 and 5 in Appendix B summarize the results obtained. It consists of the true parameters values, the posterior mean, the $95 \%$ high posterior density credibility interval and the $Z$-scores for a test to the Markov chain convergence (Geweke, 1992). It is possible to verify that the intervals contains the true values and, therefore, that the procedures developed generate trustworthy estimates for the model parameters. Once verified this capability, the next step is a real data application.

\subsection{Broadband Access Data}

The telecommunication sector is experiencing a deep changing regarding the introduction of new technologies. Whereas that context, it is highlighted the broadband diffusion, and therefore the possibility of transferring data in high speed. Academic researches point out that the advances on the availability of such services, the expansion to the access of communication and information technology cause consequences in the economic development in different ways: growth, creation of job opportunities, productivity increase and innovation.

Czernich, Falck, Kretschmer, and Woessmann (2011) estimate the effects of broadband infrastructure in the economic growth by using a panel data with observations of 25 countries from OECD through the years of 1996 and 2007. As a conclusion of the research, the authors have stated that the increase of $10 \%$ in the broadband access implies in the increase of the domestic product between $0.9 \%$ and $1.5 \%$. Koutroumpis (2009), on the other hand, concluded that the same increase in the broadband access provides an affix of $0.25 \%$ in the product by observing a twenty-two-country database. Whereas a wider database with 66 countries of high income, Zhen-Wei Qiang, Rossoto, and Kimura (2009) outline that the increase in the same $10 \%$ of the reach implies in an increase of $1.21 \%$ in the product per capita.

However, Katz (2012) highlights that the consequences in economy do not occur uniformly within the distinct sectors and, in the way, within the different countries. That author establish that, in the case of fully productivity of the 
factors, the effect is perceived more strongly in intense industry of information technology. The growth in the efficiency is also noticed more relevantly in sectors with high expense in transaction, for instance the financial sector, or intensive labor, such as tourism. Thus, in economies with more intensive use of technology, the broadband expansion presents higher impacts in the economic growth.

In this paper, based on the structure described by Roller and Waverman (2001), in which the product of an economy $(\mathbf{y})$ is generated by a production function in which the broadband services $(\mathbf{x})$ represent one more input besides the capital and the labor $\left(\mathbf{x}^{*}\right)$, the multivariate IV spatial regression model introduced in section 2 is applied to a base with observations of 5,564 Brazilian counties in the year of 2007 . Thus, supported by the content previously described in this section, it is established:

$$
\begin{aligned}
& \mathbf{y}=\text { added values from the agricultural, industrial and service } \\
& \text { sectors; } \\
& \mathbf{x}^{*}=\text { amount of employment relationship }\left(\beta_{1}\right) \text {, Molsa Família } \\
& \text { program }\left(\beta_{2}\right) \text {, municipal revenue }\left(\beta_{3}\right) \text {, continuing provi- } \\
& \text { sion benefits }\left(\beta_{4}\right) \text {, social security with the exception of } \\
& \text { continuing provision benefits }\left(\beta_{5}\right) \text {; } \\
& \mathbf{x}=\text { density of access to broadband }\left(\beta_{6}\right) \text {. }
\end{aligned}
$$

All variables are in logarithmic scale. Thence, the $\boldsymbol{\beta}$ coefficients are interpreted as elasticity coefficients, it means that they indicate the percentage change that will occur on $\mathbf{y}$ when the covariates changes one percent.

The methodology of instrumental variables is used, in this case, to model the density of access to broadband variable and treat the problem of reverse causality between itself and the added values. Therefore, the instrumental variables are specified as $\mathrm{z}=$ "density of access to the service of fixed telephony $\left(\delta_{1}\right)$, density of access to the internet between $512 \mathrm{kbps}$ and $2 \mathrm{Mbps}\left(\delta_{2}\right)$ ". The idea of using those variables as instruments lies in the fact that there is no more relevant impact caused by them on the economy even though it was relevant in the past. However, the fixed telephony and the speed band between $512 \mathrm{kbps}$ and $2 \mathrm{Mbps}$ infrastructures would currently serve as a mean for the expansion of broadband services.

Whereas the estimation process to the proposed model, the hyperparameters set mentioned above was defined as: $\boldsymbol{d}_{0}=\mathbf{0}_{(\mathbf{3} \times \mathbf{1})}, \mathbf{D}_{0}=\mathbf{I}_{3} 10^{3}, \mathbf{b}_{0}=\mathbf{0}_{(\mathbf{7} \times \mathbf{3})}$, 
$\mathbf{B}_{0}=\mathbf{I}_{7} 10^{3}, \nu_{0}=5$ and $\boldsymbol{\Sigma}_{\mathbf{0}}=\mathbf{I}_{\mathbf{4}}$, which corresponds to non-informative priors specification. 50,000 iterations of the algorithm MCMC were executed, in which the first 20,000 were discarded like the warming period, and then the following 30,000 were saved. In order to reduce the autocorrelation within the successive values of the simulated chain, it was required a thin equals to 30 . Finally, the estimations a posteriori were performed based on a sample size of 1,000.

Tables 1, 2, 3 summarize the results obtained on the estimation process and all of them contains the posterior mean estimates of the parameters in addition to their corresponding $95 \%$ high posterior density credibility interval and the $Z$-scores for a test to the Markov chain convergence (Geweke, 1992). Considering Table 1 and Figure 1, focusing the attention to parameter $\phi$, it is possible to state that the spatial component has different effects between the sectors and non zero effect for all variables. It is interesting to highlight that several agricultural properties are situated in more than one county and the relevance of the spatial influence on this sector is reflected by $\phi$ posterior mean 0.6658 as the highest one.

The instrumental variable coefficients $\left(\beta_{6}\right)$ have non zero effect for all the three sectors under analysis, since the credibility interval is away from zero. Additionally, the estimates are in accordance with the observations outlined by Katz (2012), i.e., the broadband access has different effects on the different gross domestic product (GDP) sectors and it impacts more the ones in which the intensive use of technology is demanded. The result for the agricultural sector can be interpreted as, in the majority of the Brazilian counties, the production is concentrated in small properties, the broadband access is not reverted in economic growth.

Based on the credibility intervals presented on Table 2, it is possible to affirm that both instruments are significant and that they are not weak instruments. Table 3, on its turn, introduces covariance matrix estimation results and give some outcomes about the instrumental variable endogeneity degree $(\rho)$. In this paper, the called "weak endogeneity in the frequentist sense" or conditional endogeneity is taken for granted, it means that the case that $\Sigma$ is integrated out is not examined. Hence, it is not difficult to conclude that $\rho=28 \%$ for the agricultural sector variable, $\rho=-6 \%$ for the industrial sector variable and $\rho=-39 \%$ for the service sector variable. 
Table 1. Estimation results for the parameters $\beta$ and $\phi$. First row: posterior mean. Second row: $95 \%$ high posterior density credibility interval. Third row: convergence test $Z$-scores.

\begin{tabular}{|c|c|c|c|}
\hline Parameters & AGRICULTURAL & INDUSTRIAL & SERVICE \\
\hline$\beta_{0}$ & $\begin{array}{c}-0.3784 \\
(-1.172,0.351) \\
0.0820\end{array}$ & $\begin{array}{c}-3.3664 \\
(-3.9528,-2.6568) \\
0.9992\end{array}$ & $\begin{array}{c}-4.3605 \\
(-4.9989,-3.6848) \\
-0.3604\end{array}$ \\
\hline$\beta_{1}$ & $\begin{array}{c}0.4095 \\
(0.3707,0.4569) \\
0.7592\end{array}$ & $\begin{array}{c}0.9999 \\
(0.9622,1.0327) \\
-0.2647\end{array}$ & $\begin{array}{c}0.5622 \\
(0.5380,0.5824) \\
0.3128\end{array}$ \\
\hline$\beta_{2}$ & $\begin{array}{c}0.0272 \\
(-0.004,0.0652) \\
-0.0914\end{array}$ & $\begin{array}{c}-0.1491 \\
(-0.1802,-0.119) \\
-1.0351\end{array}$ & $\begin{array}{c}0.1927 \\
(0.1695,0.2137) \\
0.2120\end{array}$ \\
\hline$\beta_{3}$ & $\begin{array}{c}0.0319 \\
(-0.0177,0.0861) \\
-0.9744\end{array}$ & $\begin{array}{c}0.3625 \\
(0.3196,0.4072) \\
0.7512\end{array}$ & $\begin{array}{c}0.1992 \\
(0.1713,0.223) \\
-0.1864\end{array}$ \\
\hline$\beta_{4}$ & $\begin{array}{c}0.0256 \\
(0.0069,0.0419) \\
-1.1986\end{array}$ & $\begin{array}{c}0.0065 \\
(-0.0095,0.0215) \\
-0.3931\end{array}$ & $\begin{array}{c}0.0032 \\
(-0.0065,0.0121) \\
-0.7163\end{array}$ \\
\hline$\beta_{5}$ & $\begin{array}{c}-0.0127 \\
(-0.0381,0.0144) \\
0.1239\end{array}$ & $\begin{array}{c}-0.0143 \\
(-0.0385,0.0066) \\
-0.4963\end{array}$ & $\begin{array}{c}0.0246 \\
(0.0088,0.0365) \\
-1.0595\end{array}$ \\
\hline$\beta_{6}$ & $\begin{array}{c}-0.1595 \\
(-0.1978,-0.1248) \\
-0.7758\end{array}$ & $\begin{array}{c}0.1407 \\
(0.1092,0.1693) \\
1.0099\end{array}$ & $\begin{array}{c}0.2912 \\
(0.2725,0.3087) \\
-0.4336\end{array}$ \\
\hline$\phi$ & $\begin{array}{c}0.6658 \\
(0.6369,0.6952) \\
0.5009\end{array}$ & $\begin{array}{c}0.1606 \\
(0.1355,0.1870) \\
-1.2299\end{array}$ & $\begin{array}{c}0.3890 \\
(0.3378,0.4319) \\
0.3868\end{array}$ \\
\hline
\end{tabular}

Table 2. Estimation results for the parameter $\boldsymbol{\delta}$ and $\gamma$. First row: posterior mean. Second row: $95 \%$ high posterior density credibility interval. Third row: convergence test $Z$-scores.

\begin{tabular}{c|c|c|c|c|c}
\hline Parameters & Estimates & Parameters & Estimates & Parameters & Estimates \\
\hline \multirow{2}{*}{$\gamma$} & -2.7884 & & 0.6717 & 0.4780 \\
& $(-2.9173,-2.6696)$ & $\delta_{1}$ & $(0.6449,0.7034)$ & $\delta_{2}$ & $(0.4672,0.4886)$ \\
& -0.7384 & & 0.6258 & -0.0958 \\
\hline
\end{tabular}



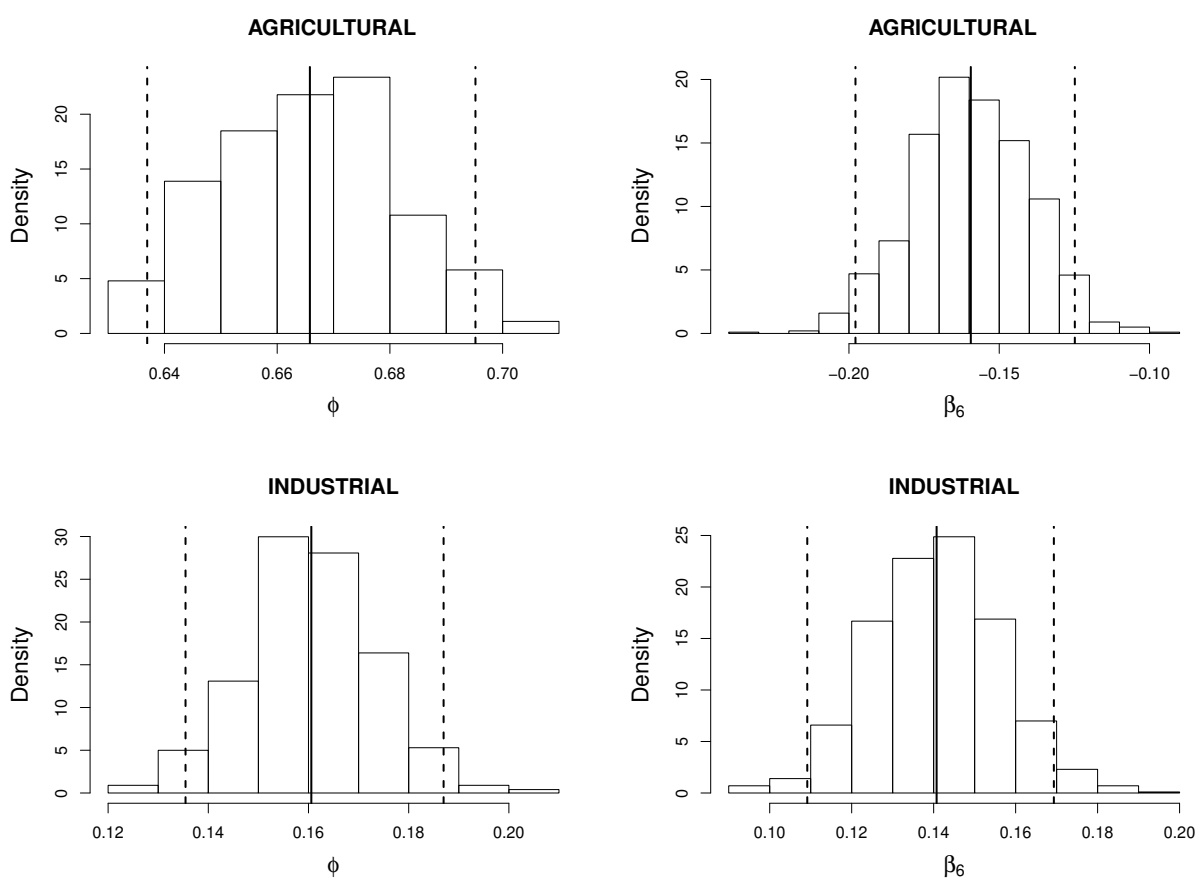

SERVICE

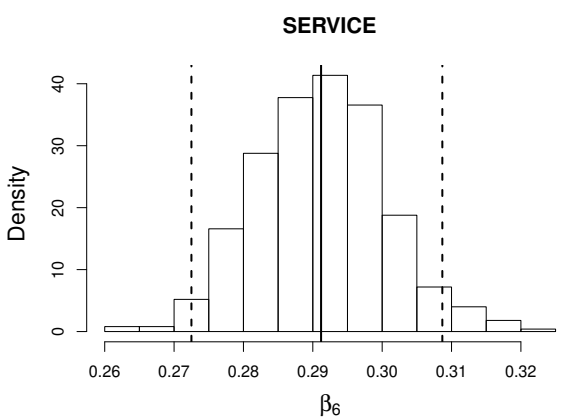

Figure 1. Economic sectors $\phi$ and $\boldsymbol{\beta}_{6}$ full conditional samples. The dashed lines represent the credibility intervals and the solid lines, the posterior means. 
Table 3. Estimation results for the covariance matrix $\boldsymbol{\Sigma}$. First row: posterior mean. Second row: $95 \%$ high posterior density credibility interval. Third row: convergence test $Z$-scores.

\begin{tabular}{c|c|c|c}
\hline Parameters & Estimates & Parameters & Estimates \\
\hline \multirow{2}{*}{$\Sigma_{11}$} & 0.7164 & & -0.0458 \\
& $(0.6907,0.7433)$ & $\Sigma_{13}$ & $(-0.077,-0.0163)$ \\
& -1.4076 & & -0.3040 \\
\hline \multirow{2}{*}{$\Sigma_{22}$} & 1.2012 & & -0.1796 \\
& $(1.1475,1.2516)$ & $\Sigma_{14}$ & $(-0.2007,-0.1599)$ \\
& 0.7381 & & -0.2034 \\
\hline \multirow{2}{*}{$\Sigma_{33}$} & 0.7738 & & 0.0728 \\
& $(0.7454,0.8051)$ & $\Sigma_{23}$ & $(0.0444,0.0975)$ \\
& -0.6872 & & -0.0030 \\
\hline \multirow{2}{*}{$\Sigma_{44}$} & 0.3054 & & 0.0741 \\
& $(0.2809,0.328)$ & $\Sigma_{24}$ & $(0.0553,0.0918)$ \\
& 0.3671 & & -1.6121 \\
\hline \multirow{2}{*}{$\Sigma_{12}$} & 0.2641 & \multirow{2}{*}{$\Sigma_{34}$} & 0.2106 \\
& $(0.2227,0.302)$ & & $(0.1929,0.2266)$ \\
& 0.0668 & & -1.1674 \\
\hline
\end{tabular}

\section{Conclusion}

This paper discusses, from a Bayesian perspective, a class of potentially useful extensions of the instrumental variables regression. First a multivariate extension of the methodology presented by Rossi et al. (2005) is introduced. Then, a spatial variation is incorporated in the fixed part of the model. The main advantage of the Bayesian approach is the possibility to explore the posterior distribution of all model parameters. Instead, other approaches only a point estimation, typically obtained after asymptotic results.

The developed inferential procedures are illustrated in the context of an economic application. The impact of access to broadband in the different economic sectors was measured through a data base with observations of 5,564 Brazilian counties in the year of 2007. The methodology proposed in this work made feasible the analysis about how the broadband investment impacts the different economic sectors. An interesting task for future research would be to analyze the behavior of the multivariate spatial IV regression in the presence of many weak instruments. Another worthwhile task would be to verify the inclusion of different spatial structures. 


\section{References}

Anselin, L. (1988). Spatial econometrics: Methods and models. Dordrecht: Kluwer.

Chamberlain, G. (2007). Decision theory applied to an instrumental variables model. Econometrica, 75(3), 609-652. http://dx.doi.org/10.1111/j.1468 $-0262.2007 .00764 . \mathrm{x}$

Conley, T. G., Hansen, C. B., McCulloch, R. E., \& Rossi, P. E. (2008). A semi-parametric Bayesian approach to the instrumental variable problem. Journal of Econometrics, 144(1), 276-305. http://dx.doi.org/10.1016/ j.jeconom.2008.01.007

Czernich, N., Falck, O., Kretschmer, T., \& Woessmann, L. (2011). Broadband infrastructure and economic growth. The Economic Journal, 121(552), 505-532. http://dx.doi.org/10.1111/j.1468-0297.2011.02420.x

Gamerman, D., \& Moreira, A. R. B. (2004). Multivariate spatial regression models. Journal of Multivariate Analysis, 91(2), 262-281. http://dx.doi .org/10.1016/j.jmva.2004.02.016

Geweke, J. (1992). Evaluating the accuracy of sampling-based approaches to the calculation of posterior moments. In J. O. Berger, A. P. Dawid, \& A. F. M. Smith (Eds.), Bayesian statistics 4 (pp. 169-193). Oxford University Press.

Katz, R. (2012, April). The impact of broadband on the economy: Research to date and policy issues (Broadband Series). International Telecommunication Union (ITU). Retrieved from https://www.itu-ilibrary.org/science-and -technology/impact-of-broadband-on-the-economy_pub/807b38cb-en

Kleibergen, F., \& Zivot, E. (2003). Bayesian and classical approaches to instrumental variable regression. Journal of Econometrics, 114(1), 29-72. http://dx.doi.org/10.1016/S0304-4076(02)00219-1

Koutroumpis, P. (2009). The economic impact of broadband on growth: A simultaneous approach. Telecommunications Policy, 33(9), 471-485. http://dx.doi.org/10.1016/j.telpol.2009.07.004

Lancaster, T. (2004). Introduction to modern Bayesian econometrics. New York: Wiley-Blackwell.

Lindley, D. V., \& El-Sayyad, G. M. (1968). The Bayesian estimation of a linear functional relationship. Journal of Royal Statistical Society, Series B, 30(1), 190-202. Retrieved from https://www.jstor.org/stable/2984471

Lopes, H. F., \& Polson, N. G. (2014). Bayesian instrumental variables: Likelihoods and priors. Econometric Reviews, 33(1-4), 100-121. http:// dx.doi.org/10.1080/07474938.2013.807146

Pace, R. K., Barry, R., Gilley, O. W., \& Sirmans, C. F. (2000). A method for spatial-temporal forecasting with an application to real estate prices. International Journal of Forecasting, 16(2), 262-281. http://dx.doi.org/ 10.1016/S0169-2070(99)00047-3 
Roller, L.-H., \& Waverman, L. (2001). Telecommunications infrastracture and economic development: A simultaneous approach. American Economic Review, 91(4), 909-923. http://dx.doi.org/10.1257/aer.91.4.909

Rossi, P. E., Allenby, G. M., \& McCulloch, R. E. (2005). Bayesian statistics and marketing. New York: Wiley.

Shaby, B. A., \& Wells, M. T. (2010). Exploring an adaptive metropolis algorithm. Retrieved from http://personal.psu.edu/bas59/papers/ adaptive_techreport.pdf

Zellner, A. (1971). An introduction to Bayesian inference in econometrics. New York: Wiley.

Zellner, A., Bauwens, L., \& van Dijk, H. K. (1988). Bayesian specification analysis and estimation of simultaneous equation models using Monte Carlo methods. Journal of Econometrics, 38(1-2), 39-72. http://dx.doi.org/ 10.1016/0304-4076(88)90026-7

Zellner, A., Tobias, J., \& Ryu, H. K. (1997). Bayesian method of moments (BMOM) analysis of parametric and semi-parametric regression models. South African Statistical Journal, 32(1), 41-69. Retrieved from https:// hdl.handle.net/10520/AJA0038271X_530

Zellner, A., \& Vandaele, W. (1975). Bayes-Stein estimators for $k$-means, regression and simultaneous equation models. In S. E. Fienberg \& A. Zellner (Eds.), Studies in Bayesian econometrics and statistics (pp. 627-653). Amsterdam: North-Holland.

Zhen-Wei Qiang, C., Rossoto, C. M., \& Kimura, K. (2009). Economic impacts of broadband. In M. A. Khalil et al. (Eds.), 2009 information and communications for development: Extending reach and increasing impact (pp. 35-50). Washington, DC: The World Bank. Retrieved from http://documents.worldbank.org/curated/en/645821468337815208/ 2009-information-and-communications-for-development-extending -reach-and-increasing-impact

\section{Appendix A Full conditional distributions}

Considering the full conditional distributions described on the equations (14)(17) as specified in subsection 3.2, the following closed form full conditional distributions are obtained:

- $(\gamma, \boldsymbol{\delta})$

$$
\begin{aligned}
p(\boldsymbol{\gamma}, \boldsymbol{\delta} \mid \cdots) \propto\left[\prod_{i=1}^{n} \exp \{\right. & \left.\left.-\frac{1}{2} \operatorname{tr}\left(\boldsymbol{\Sigma}_{1 \mid 2}\left(\tilde{\tilde{\mathbf{x}}}_{i}-\boldsymbol{\delta}^{*} \tilde{\mathbf{z}}_{i}\right)\left(\tilde{\tilde{\mathbf{x}}}_{i}-\boldsymbol{\delta}^{*} \tilde{\mathbf{z}}_{i}\right)^{\prime}\right)\right\}\right] \\
& \times \exp \left\{-\frac{1}{2} \operatorname{tr}\left(\boldsymbol{\Sigma}_{1 \mid 2}\left(\boldsymbol{\delta}^{*}-\boldsymbol{d}_{0}\right) \mathbf{D}_{0}^{-1}\left(\boldsymbol{\delta}^{*}-\boldsymbol{d}_{0}\right)^{\prime}\right)\right\},
\end{aligned}
$$


in which $\boldsymbol{\delta}^{*}=(\boldsymbol{\gamma}, \boldsymbol{\delta}), \tilde{\mathbf{z}}_{i}=\left(1, \mathbf{z}_{i}^{\prime}\right)^{\prime}$ and

$$
\tilde{\tilde{\mathbf{x}}}_{i}=\mathbf{x}_{i}-\boldsymbol{\Sigma}_{12} \boldsymbol{\Sigma}_{22}^{-1}\left[\mathbf{y}_{i}-\boldsymbol{\phi}(\mathbf{W} \mathbf{y})_{i}^{\prime}-\boldsymbol{\beta}_{1} \mathbf{x}_{i}^{*}-\boldsymbol{\beta}_{2} \mathbf{x}_{i}\right] .
$$

Hence,

$$
(\boldsymbol{\gamma}, \boldsymbol{\delta})^{\prime} \mid \boldsymbol{\beta}_{1}, \boldsymbol{\beta}_{2}, \boldsymbol{\Sigma}, \mathbf{x}, \mathbf{y}, \mathbf{z} \sim \mathcal{N}_{(l+1) \times p}\left(\boldsymbol{d}_{1}, \mathbf{D}_{1}, \boldsymbol{\Sigma}_{1 \mid 2}\right)
$$

where $\mathbf{D}_{1}^{-1}=\mathbf{D}_{0}^{-1}+\sum_{i=1}^{n} \tilde{\mathbf{z}}_{i} \tilde{\mathbf{z}}_{i}^{\prime}$ and $\mathbf{D}_{1}^{-1} \boldsymbol{d}_{1}=\mathbf{D}_{0}^{-1} \boldsymbol{d}_{0}+\sum_{i=1}^{n} \tilde{\mathbf{z}}_{i} \tilde{\tilde{\mathbf{x}}}_{i}^{\prime}$.

- $\left(\boldsymbol{\beta}_{1}, \boldsymbol{\beta}_{2}\right)$

$$
\begin{aligned}
p\left(\boldsymbol{\beta}_{1}, \boldsymbol{\beta}_{2} \mid \cdots\right) \propto\left[\prod_{i=1}^{n} \exp \{\right. & \left.\left.-\frac{1}{2} \operatorname{tr}\left(\boldsymbol{\Sigma}_{2 \mid 1}\left(\tilde{\mathbf{y}}_{i}-\boldsymbol{\beta} \tilde{\mathbf{x}}_{i}\right)\left(\tilde{\mathbf{y}}_{i}-\boldsymbol{\beta} \tilde{\mathbf{x}}_{i}\right)^{\prime}\right)\right\}\right] \\
& \times \exp \left\{-\frac{1}{2} \operatorname{tr}\left(\boldsymbol{\Sigma}_{2 \mid 1}\left(\boldsymbol{\beta}-\mathbf{b}_{0}\right) \mathbf{B}_{0}^{-1}\left(\boldsymbol{\beta}^{*}-\mathbf{b}_{0}\right)^{\prime}\right)\right\},
\end{aligned}
$$

in which $\boldsymbol{\beta}=\left(\boldsymbol{\beta}_{1}, \boldsymbol{\beta}_{2}\right), \tilde{\mathbf{x}}_{i}=\left(\mathbf{x}_{i}^{*}, \mathbf{x}_{i}\right)$ and

$$
\tilde{\mathbf{y}}_{i}=\mathbf{y}_{i}-\left[\boldsymbol{\phi}(\mathbf{W} \mathbf{y})_{i}^{\prime}+\boldsymbol{\Sigma}_{21} \boldsymbol{\Sigma}_{11}^{-1}\left(\mathbf{x}_{i}-\boldsymbol{\gamma}-\boldsymbol{\delta} \mathbf{z}_{i}\right)\right]
$$

Hence,

$$
\left(\boldsymbol{\beta}_{1}, \boldsymbol{\beta}_{2}\right)^{\prime} \mid \boldsymbol{\gamma}, \boldsymbol{\delta}, \boldsymbol{\Sigma}, \mathbf{x}, \mathbf{y}, \mathbf{z} \sim \mathcal{N}_{(r+p) \times q}\left(\mathbf{b}_{1}, \mathbf{B}_{1}, \boldsymbol{\Sigma}_{2 \mid 1}\right)
$$

where $\mathbf{B}_{1}^{-1}=\mathbf{B}_{0}^{-1}+\sum_{i=1}^{n} \tilde{\mathbf{x}}_{i} \tilde{\mathbf{x}}_{i}^{\prime}$ and $\mathbf{B}_{1}^{-1} \mathbf{b}_{1}=\mathbf{B}_{0}^{-1} \mathbf{b}_{0}+\sum_{i=1}^{n} \tilde{\mathbf{x}}_{i} \tilde{\mathbf{y}}_{i}^{\prime}$.

- $\Sigma$

$$
\begin{aligned}
p(\boldsymbol{\Sigma} \mid \cdots) \propto\left[\prod_{i=1}^{n}|\boldsymbol{\Sigma}|^{-\frac{1}{2}} \exp \left\{-\frac{1}{2} \operatorname{tr}\left(\boldsymbol{\Sigma}^{-1} V_{i} V_{i}^{\prime}\right)\right\}\right] \\
\times|\Sigma|^{-\frac{\nu_{0}+(p+q)+1}{2}} \exp \left\{-\frac{1}{2} \operatorname{tr}\left(\boldsymbol{\Sigma}_{0} \boldsymbol{\Sigma}^{-1}\right)\right\},
\end{aligned}
$$

in which $V_{i}=\left(V_{1 i}^{\prime}, V_{2 i}^{\prime}\right)^{\prime}, V_{1 i}=\mathbf{x}_{i}-\boldsymbol{\gamma}-\boldsymbol{\delta} \mathbf{z}_{i}$ and $V_{2 i}=\mathbf{y}_{i}-\boldsymbol{\phi}(\mathbf{W y})_{i}^{\prime}-$ $\boldsymbol{\beta}_{1} \mathbf{x}_{i}^{*}-\boldsymbol{\beta}_{2} \mathbf{x}_{i}$. Hence,

$$
\Sigma \mid \boldsymbol{\gamma}, \boldsymbol{\delta}, \boldsymbol{\beta}_{1}, \boldsymbol{\beta}_{2}, \mathbf{x}, \mathbf{y}, \mathbf{z} \sim \mathcal{I} \mathcal{W}\left(\nu_{1}, \boldsymbol{\Sigma}_{1}\right)
$$

where $\nu_{1}=\nu_{0}+n$ and $\boldsymbol{\Sigma}_{1}=\boldsymbol{\Sigma}_{0}+\sum_{i=1}^{n} V_{i} V_{i}^{\prime}$.

For the parameters $\phi_{k k}$ it was not possible to find closed form full conditional distributions. In this case, a Metropolis-Hastings step was required. As a proposal distribution it was adopted $\phi_{k k}^{\text {novo }} \sim \mathcal{N}_{(0,1)}\left(\phi_{k k}, c_{\phi_{k k}}\right), \mathcal{N}_{A}$ truncated 
normal on the interval $A$, where $c_{\phi_{k k}}$ is an adaptive parameter as introduced by Shaby and Wells (2010).

\section{Appendix B Synthetic Data Estimates}

Table 4. Estimation results for the artificial data. First row: posterior mean. Second row: $95 \%$ high posterior density credibility interval. Third row: convergence test $Z$-scores.

\begin{tabular}{|c|c|c|c|c|c|c|c|c|}
\hline Parameters & TRUE & Estimates & Parameters & TRUE & Estimates & Parameters & TRUE & Estimates \\
\hline$\beta_{1,01}$ & 0.5 & $\begin{array}{c}-0.3985 \\
(-2.3357,1.5882) \\
-0.9245\end{array}$ & $\beta_{1,02}$ & 3.4 & $\begin{array}{c}4.2339 \\
(1.6156,6.7417) \\
0.2021 \\
\end{array}$ & $\beta_{1,03}$ & 2.7 & $\begin{array}{c}2.5468 \\
(-0.3931,6.0474) \\
0.3563 \\
\end{array}$ \\
\hline$\beta_{1,11}$ & 6 & $\begin{array}{c}5.9968 \\
(5.8911,6.1189) \\
-0.2576\end{array}$ & $\beta_{1,12}$ & 1.5 & $\begin{array}{c}1.4813 \\
(1.3932,1.5736) \\
-0.7859\end{array}$ & $\beta_{1,13}$ & 1.2 & $\begin{array}{c}1.1405 \\
(1.0164,1.2528) \\
-0.6109\end{array}$ \\
\hline$\beta_{1,21}$ & 1.8 & $\begin{array}{c}1.5473 \\
(0.0064,2.8854) \\
0.1727\end{array}$ & $\beta_{1,22}$ & 2.1 & $\begin{array}{c}1.7846 \\
(1.0423,2.5211) \\
0.8549\end{array}$ & $\beta_{1,23}$ & -2.3 & $\begin{array}{c}-2.8391 \\
(-3.8753,-1.8297) \\
-0.0044\end{array}$ \\
\hline$\beta_{2,11}$ & 2 & $\begin{array}{c}1.7872 \\
(1.4108,2.2434) \\
-0.6448 \\
\end{array}$ & $\beta_{2,12}$ & -3 & $\begin{array}{c}-2.9434 \\
(-3.4231,-2.4468) \\
0.0673\end{array}$ & $\beta_{2,13}$ & -4 & $\begin{array}{c}-4.1733 \\
(-4.7416,-3.4391) \\
-0.4058\end{array}$ \\
\hline$\beta_{2,21}$ & 1 & $\begin{array}{c}1.1691 \\
(0.8547,1.4657) \\
0.6917 \\
\end{array}$ & $\beta_{2,22}$ & 0.7 & $\begin{array}{c}0.6675 \\
(0.2941,1.0029) \\
-0.0626 \\
\end{array}$ & $\beta_{2,23}$ & 3.7 & $\begin{array}{c}3.8331 \\
(3.2951,4.2659) \\
0.3979 \\
\end{array}$ \\
\hline$\phi_{11}$ & 0.2 & $\begin{array}{c}0.2008 \\
(0.1573,0.2430) \\
0.0992\end{array}$ & $\phi_{22}$ & 0.5 & $\begin{array}{c}0.4831 \\
(0.4282,0.5346) \\
0.0066\end{array}$ & $\phi_{33}$ & 0.8 & $\begin{array}{c}0.7982 \\
(0.7437,0.8524) \\
-0.7315\end{array}$ \\
\hline$\gamma_{1}$ & -2.5 & $\begin{array}{c}-2.6819 \\
(-2.8698,-2.4771) \\
0.6019\end{array}$ & $\delta_{11}$ & -2.3 & $\begin{array}{c}-2.2817 \\
(-2.3656,-2.1972) \\
-1.1518\end{array}$ & $\delta_{21}$ & 1.2 & $\begin{array}{c}1.2571 \\
(1.2000,1.3112) \\
0.2358 \\
\end{array}$ \\
\hline$\gamma_{2}$ & 2.1 & $\begin{array}{c}1.9437 \\
(1.6133,2.2739) \\
1.2211\end{array}$ & $\delta_{12}$ & -2.8 & $\begin{array}{c}-2.7657 \\
(-2.9061,-2.6412) \\
-1.0409\end{array}$ & $\delta_{22}$ & 1.8 & $\begin{array}{c}1.8481 \\
(1.7656,1.9291) \\
-0.3177\end{array}$ \\
\hline
\end{tabular}


Table 5. Estimation results for the main diagonal of the covariance matrix. First row: posterior mean. Second row: $95 \%$ high posterior density credibility interval. Third row: convergence test $Z$-scores.

\begin{tabular}{|c|c|c|}
\hline Parameters & TRUE & $95 \%$ \\
\hline$\Sigma_{11}$ & 4 & $\begin{array}{c}4.8367 \\
(3.9082,6.0191) \\
-0.3381\end{array}$ \\
\hline$\Sigma_{22}$ & 9 & $\begin{array}{c}9.5462 \\
(8.2098,10.8747) \\
-0.3565\end{array}$ \\
\hline$\Sigma_{33}$ & 16 & $\begin{array}{c}17.1189 \\
(14.9272,19.4248) \\
-0.9064\end{array}$ \\
\hline$\Sigma_{44}$ & 4 & $\begin{array}{c}3.5667 \\
(3.1306,4.0287) \\
0.0850\end{array}$ \\
\hline$\Sigma_{55}$ & 9 & $\begin{array}{c}8.2902 \\
(7.3125,9.4366) \\
-1.4937\end{array}$ \\
\hline
\end{tabular}

